\title{
An adapted group-dilation anisotropic multifractal formalism for functions
}

Anouar Ben MABROUK

Computational Mathematics Laboratory, Department of Mathematics, Faculty of

Sciences. 5000 Monastir, Tunisia

E-mail: anouar.benmabrouk@issatso.rnu.tn

Received September 11, 2007; Accepted in Revised Form November 15, 2007

\begin{abstract}
Anisotropic phenomena can be observed almost everywhere in nature. This makes them important subjects for theoretical and experimental studies. In this work, we focus on the study of anisotropic quasi-self-similar signals. It holds that the classical multifractal formalism in all its formulations does not hold for this class. We then use an homogeneous norm introduced by Calderon and Torchinsky to check the validity of an adapted anisotropic multifractal formalism for these signals. Our techniques are based on group theory combined with wavelet characterization of anisotropic function spaces. We then show the efficiency of anisotropic wavelets in detecting singularities.
\end{abstract}

\section{Introduction}

Suppose that one has an object that depends on several parameters, for examples, a moving solid, and that the on these parameters differs from one to another. We say simply that such objects follow a nonisotropic motion. The anisotropy is then the fact of depending differently on given parameters such as the coordinates of a physical basis. It can be observed nearly everywhere in natural phenomena such as trees, lung structure and wind. As a consequence, the concept of anisotropy has assumed an importance and thereafter has been a subject of interest for the researchers in theoretical mathematics as well as in physical and data-processing practice. This work lies within this whole topic and deals with some studies of anisotropic phenomena and their treatments with wavelet analysis. There are many motivations behind this work. Some of them are cited in our work [8]. We hereafter cite some other interesting motivations.

- Anisotropy can appear in seismology. This phenomenon has been noticed in several studies. Researchers guess that the observed seismic anisotropy is due to strain induced lattice preferred orientation of olivine caused by the amalgamation and deformation of blocks.

- Atmospheric phenomena. It is known now from experimental physical studies that the horizontal spectrum of wind involves scaling but not with the same scale law as the vertical one. This fact led physicists to think that the anisotropy is not independent of the gravity as was once thought. Geophysical turbulence is strongly anisotropic. This is essentially due to the gravity effects in all scales. 
- The correlations between the topography and gravity anomalies cause significant anisotropy. Indeed, the flexural rigidity of the lithosphere is usually assumed to be isotropic. However, it has been shown recently that the coherence increases in one direction compared to the azimuthal average, which leads to the anisotropy.

- One can also observe anisotropy phenomena in rough surfaces used as an instrument for simulating the topography of engineering surfaces. Rough surfaces play an important role in several areas of engineering and science such as design of superconductors, machine design, materials science, scattering of electromagnetic waves, surface contact and wear mechanics and tribology.

- Anisotropy can be observed in the diffusion into fibrous anisotropic structures exhibiting a variety of crossover phenomena.

- An important motivation of our work is related to group theory. This is motivated by the fact that wavelets, have since their appearance, been strongly related to group theory. Recall that from the starting ideas by Grossmann, Morlet, and others, wavelets are certain coherent states associated with the affine group on the line generated by dilation and translation. The action of the scale parameter $a$ or $j$ referred to the frequency on the mother wavelet $\psi$ which is a dilation-contraction and the action of the position parameter $b$ yield a coherent state associated to the affine group $a x+b$ of the real line. The present work is based on a group action on wavelets. We use some special subgroups of the matrix group $G L(n)$ to adopt and check some coherent situations associated to the anisotropic behaviors of an important class of signals. It consists here of an affine-like group.

More about these motivations and other applications about wavelets, anisotropy and their relations to group theory, anisotropic deformations, and group deformation can be found in $[2],[4],[5],[6],[7],[8],[9],[10],[12],[13],[14],[15],[17],[18],[23],[25],[26],[27],[29]$ and the references therien.

In [6], [7], [8], [9], [10] and [12], some special types of singularities for anisotropic quasiself-similar functions and distributions in both the linear and nonlinear cases have been studied. The idea was to define a new type of regularity taking into account the anisotropic behavior. Some new variants of the spectrum of singularities have been introduced in order to be adequate for the new type of singularities. The principal idea for computing the spectra was to adapt some variants of the Hausdorff measure and dimension. A return to the multifractal formalism due to Arneodo et al [3] have been carried out. The principal idea behind the cited references was the inadequacy of the multifractal formalism for anisotropic self-similar functions.

In this paper, we revisit this problem and we propose to undertake a study in anisotropic function spaces. We then use an homogeneous norm due to Calderon and Torchinsky to obtain good results. We intend that such a norm interacts well with the anisotropic contractions. Then we show how one may modify the known formulations in order to adapt them to a large class of nonhomogenous functions. We now recall some preliminaries on the homogeneous norm. For backgrounds, we refer to [12], [15], [19], [20], [24] and [28].

For $r>0$, consider the group of matrices $A_{r}=\operatorname{diag}\left(r^{d_{1}}, r^{d_{2}}, \ldots, r^{d_{m}}\right)$ where the $d_{j}$ 's are real parameters such that $d_{1}=1 \leq d_{2} \leq \ldots \leq d_{m}$. Consider for $x \in \mathbb{R}^{m} \backslash 0$ fixed the 
function

$$
\varphi_{x}(r)=\left\|A_{r}^{-1} x\right\|=\sqrt{r^{-2 d_{1}} x_{1}^{2}+r^{-2 d_{2}} x_{2}^{2}+\ldots+r^{-2 d_{m}} x_{m}^{2}} .
$$

It is a continuous non increasing function and it maps the interval $] 0,+\infty[$ into itself. So, there exists a unique real number $r=r(x)$ for which $\varphi_{x}(r)=1$. We define then the homogeneous norm of $x \in \mathbb{R}^{m}$ by

$$
\rho(x)=\left\{\begin{array}{cc}
r(x) & , x \neq 0 \\
0 & , x=0 .
\end{array}\right.
$$

Some basic properties of the homogeneous norm will be exposed in the appendix at the end of the paper. We adopt now some notations which will be useful later on. For a multi-index $i=\left(i_{1}, i_{2}, \ldots, i_{m}\right) \in \mathbb{N}^{m}$, we denote its length

$$
|i|=i_{1}+i_{2}+\ldots+i_{m} .
$$

We denote also

$$
\partial^{i}=\partial_{x_{1}}^{i_{1}} \partial_{x_{2}}^{i_{2}} \ldots \partial_{x_{m}}^{i_{m}}
$$

the partial differential operator according to $i$ and

$$
d(i)=d_{1} i_{1}+d_{2} i_{2}+\ldots+d_{m} i_{m}
$$

the order of such an operator and finally we write

$$
x^{i}=x_{1}{ }^{i_{1}} x_{2}{ }^{i_{2}} \ldots x_{m}{ }^{i_{m}}, \forall x \in \mathbb{R}^{m} .
$$

We recall that if $P(x)=\sum_{i} a_{i} x^{i}$ is an homogeneous real valued polynomial on $\mathbb{R}^{m}$, its homogeneous degree is $d_{\rho}(P)=\max \left\{d(i) ; a_{i} \neq 0\right\}$.

We will now give the modified versions of Hölder regularity and the wavelet transform.

Definition 1. Let $\alpha>0$ and $x_{0} \in \mathbb{R}^{m}$. A real valued function $F: \mathbb{R}^{m} \rightarrow \mathbb{R}$ is said to be $\rho$-regular of order $\alpha$ at $x_{0}$ if there exists a constant $C$, a polynomial $P$ with homogeneous degree less than $[\alpha]$ and a neighborhood $\mathcal{V}\left(x_{0}\right)$ satisfying

$$
\left|F(x)-P\left(x-x_{0}\right)\right| \leq C \rho\left(x-x_{0}\right)^{\alpha}, \quad \forall x \in \mathcal{V}\left(x_{0}\right) .
$$

We say also that $F$ has an $\alpha$ - $\rho$-singularity at $x_{0}$ and we write $F \in \mathcal{C}_{\rho}^{\alpha}\left(x_{0}\right)$. The global space of $\alpha$ - $\rho$-singularities can be defined if (1.1) holds for all $x, x_{0} \in \mathbb{R}^{m}$ with a constant $C$ being uniform. We write $F \in \mathcal{C}_{\rho}^{\alpha}\left(\mathbb{R}^{m}\right)$.

The $\rho$-singularity exponent or the $\rho$-Hölder exponent is defined by

$$
\alpha_{\rho, F}\left(x_{0}\right)=\sup \left\{\alpha ; \quad F \in \mathcal{C}_{\rho}^{\alpha}\left(x_{0}\right)\right\} .
$$

Consider now two functions $\varphi$ and $\psi$ in the Schwartz class $\mathcal{S}\left(\mathbb{R}^{m}\right)$ such that

(i) $\widehat{\varphi}$ has compact support disjoint from 0 .

(ii) $\psi$ is supported in $|x| \leq 1$ and with vanishing moments. 
(iii) For all $x \in \mathbb{R}^{m}, x \neq 0$, we have

$$
\int_{0}^{+\infty} \widehat{\varphi}\left(A_{r} x\right) \widehat{\psi}\left(A_{r} x\right) \frac{d r}{r}=1
$$

Let $x^{d}=\left(2^{d_{1}}, 2^{d_{2}}, \ldots, 2^{d_{m}}\right), \varphi^{d}(x)=\varphi\left(x-x^{d}\right)$ and $\psi^{d}(x)=\psi\left(x+x^{d}\right)$. For $a>0$ denote

$$
\varphi_{a}^{d}(x)=\frac{1}{a^{D}} \varphi^{d}\left(A_{a}^{-1} x\right) \quad \text { and } \quad \psi_{a}^{d}(x)=\frac{1}{a^{D}} \psi^{d}\left(A_{a}^{-1} x\right)
$$

where $D=d_{1}+d_{2}+\ldots+d_{m}$. The anisotropic wavelet transform of $F$ at the scale $a$ and the position $b$ will be

$$
C_{\rho, F}(a, b)=\left(F * \varphi_{a}^{d}\right)(b)=\frac{1}{a^{D}} \int_{\mathbb{R}^{m}} F(x) \varphi^{d}\left(A_{a}^{-1}(x-b)\right) d x .
$$

The following result is an analog of the classical wavelet characterization of regularity. It characterizes the $\rho$-Hölder regularity by means of anisotropic wavelets (See [12] for a proof).

Proposition 1. 1-F $\in \mathcal{C}_{\rho}^{\alpha}(\mathbb{R})$ if and only if $\left|C_{\rho, F}(a, b)\right| \leq C a^{\alpha}$. 2-If $F$ has an $\alpha$ - $\rho$-singularity at $x_{0}$ then

$$
\left|C_{\rho, F}(a, b)\right| \leq C a^{\alpha}\left(1+\frac{\rho\left(b-x_{0}\right)}{a}\right)^{\alpha} .
$$

3-If the inequality in 2 holds and if $F \in \mathcal{C}_{\rho}^{\varepsilon}(\mathbb{R})$ for some $\varepsilon>0$, there exists a polynomial $P$ such that if $\rho\left(x-x_{0}\right) \leq 1 / 2$,

$$
\left|F(x)-P\left(x-x_{0}\right)\right| \leq C \rho\left(x-x_{0}\right)^{\alpha} \log \left(\frac{1}{\rho\left(x-x_{0}\right)}\right) .
$$

The goal of the multifractal analysis of functions is to determine their pointwise and uniform Hölder exponent and to compute the Hausdorff dimension of the singularities set. In general such a problem is no longer obvious. To find a relation between the Hölder exponent and the Besov one is not simple. Whereas for certain cases, like selfaffine signals, the wavelet coefficients inherit the characters of the signal. If $F: \mathbb{R}^{m} \rightarrow \mathbb{R}$ satisfies $F(x)=\lambda F(r x)$ for all $x$ and for some $\lambda$ and $r$, then its wavelet transform satisfies

$$
C_{a, b}(F)=\lambda C_{r a, r b}(F) \quad \forall a>0 \text { and } \forall b \in \mathbb{R}^{m} .
$$

These relations allow the estimation of the size of the wavelet transform $C_{a, b}(F)$ everywhere.

The multifractal formalism is a mathematical formula that computes the Hausdorff dimension of the singularity set. In the majority of cases, it seems impossible to do such a computation from the mathematical definition of the Hausdorff dimension. So, one tries to extract suitable quantities from the studied object to simplify the problem. In some cases the computation of the spectrum of singularities is related to Besov exponents, to overage quantities based on the modules of continuity or wavelet transform. We will not recall here these notions but we will give their analogs in the anisotropic case. We say that 
a function $F$ belongs to the homogeneous anisotropic Besov space $\mathcal{B}_{\rho, p}^{s, \infty}\left(\mathbb{R}^{m}\right)$ for $s \in \mathbb{R}$ and $p>0$ if for $a$ small enough one has

$$
\int\left|C_{\rho, F}(a, b)\right|^{p} d b \leq C a^{s p}
$$

The homogeneous Besov exponent of $F$ will be

$$
\eta_{\rho}(p)=\sup \left\{s ; \quad F \in \mathcal{B}_{\rho, p}^{s, \infty}\left(\mathbb{R}^{m}\right)\right\}=\liminf _{a \rightarrow 0} \frac{\log \int\left|C_{\rho, F}(a, b)\right|^{p} d b}{\log a} .
$$

We now recall the modified Hausdorff measure and dimension. For a subset $U$ of $\mathbb{R}^{m}$, the $\rho$-diameter of $U$ is

$$
\rho(U)=\sup \{\rho(x-y) ; \quad x, y \in U\} .
$$

The $\rho$-Hausdorff measure is defined for all $E \subseteq \mathbb{R}^{m}$ and $\alpha \geq 0$ by

$$
\mathcal{H}_{\rho}^{\alpha}(E)=\lim _{\varepsilon \downarrow 0}\left(\inf \left\{\sum_{i} \rho\left(U_{i}\right)^{\alpha} ; \quad E \subseteq \bigcup_{i} U_{i}, \rho\left(U_{i}\right) \leq \varepsilon\right\}\right)
$$

and the $\rho$-Hausdorff dimension of $E$ is

$$
\operatorname{dim}_{\rho}(E)=\inf \left\{\alpha ; \mathcal{H}_{\rho}^{\alpha}(E)=0\right\} .
$$

Finally, we define the anisotropic $\alpha$ - $\rho$-singularities set

$$
E_{\rho}(\alpha)=\left\{x ; \quad \alpha_{\rho, F}(x)=\alpha\right\}
$$

and the $\rho$-spectrum of singularities $d_{\rho}(\alpha)=\operatorname{dim}_{\rho}\left(E_{\rho}(\alpha)\right)$. The anisotropic multifractal formalism affirms that

$$
d_{\rho}(\alpha)=\inf _{p}\left(\alpha p-\eta_{\rho}(p)+D\right) .
$$

In this paper we propose to check such a formalism for the class of anisotropic quasi-selfsimilar functions.

\section{Main results}

Let $\Omega$ be a bounded domain in $\mathbb{R}^{m}$ and $k>0$. Let also $\left.\mu_{1}, \mu_{2} \in\right] 0,1[$ and consider some contractions $T_{j}(x)=A_{\mu_{j}}(x)+B_{j}, j=1,2$ satisfying

$$
T_{j}(\Omega) \subset \Omega \quad \text { and } \quad T_{i}(\Omega) \cap T_{j}(\Omega)=\emptyset \quad \text { whenever } i \neq j .
$$

Consider two sequences $\left(\lambda_{j}^{n}\right)_{n}, j=1,2$ of numbers uniformly bounded in $] 0,1[$. That is,

$$
\exists a, b ; \quad 0<a \leq\left|\lambda_{j}^{n}\right| \leq b<1, \forall n, j=1,2 .
$$

In what follows we will denote for a multi-index $i=\left(i_{1}, i_{2}, \ldots, i_{n}\right) \in\{1,2\}^{n}$,

$$
\lambda_{i}=\lambda_{i_{1}}^{1} \lambda_{i_{2}}^{2} \ldots \lambda_{i_{n}}^{n} \quad \text { and } \quad \mu_{i}=\mu_{i_{1}} \mu_{i_{2}} \ldots \mu_{i_{n}} .
$$


We will write also

$$
T_{i}=T_{i_{n}} \circ \ldots \circ T_{i_{1}}, \quad x_{i}=T_{i}(0) \quad \text { and } \quad \Omega_{i}=T_{i}(\Omega) .
$$

Define for $j \in \mathbb{N}$ and $L$ large enough the sets

$$
B_{j}=\left\{i ; 2^{-j} \leq \mu_{i}<2^{1-j}\right\} \quad \text { and } \quad B_{j}(x)=\left\{i \in B_{j} ; \rho\left(x-x_{i}\right) \leq L 2^{-j}\right\}
$$

where $x_{i}=T_{i}(0)$. We recall that $B_{j}(x)$ has finite cardinality independently of $x$ and $j$. Denote

$$
\alpha_{\text {min }}=\liminf _{j \rightarrow+\infty}\left(\inf _{i \in B_{j}} \frac{\log \left|\lambda_{i}\right|}{\log \mu_{i}}\right) \quad \text { and } \quad \alpha_{\max }=\limsup _{j \rightarrow+\infty}\left(\sup _{i \in B_{j}} \frac{\log \left|\lambda_{i}\right|}{\log \mu_{i}}\right) .
$$

Finally, we denote by $K$ the unique non empty $T$-invariant compact. That is

$$
K=T_{1}(K) \cup T_{2}(K)=\bigcap_{n \geq 0} \bigcup_{|i|=n} T_{i}(\Omega) .
$$

The anisotropic quasi-self-similar functions were introduced in [6] and they were defined by means of the series

$$
F(x)=\sum_{n \geq 0} \sum_{|i|=n} \lambda_{i} g\left(T_{i}^{-1}(x)\right)
$$

where $g$ is a $\mathcal{C}^{k+1}$ real valued function with compact support on $\Omega$ and where we set as a convention $g\left(T_{i}^{-1}(x)\right)=0$ for all $x \notin \Omega_{i}$. The function $F$ can be written in a different way

$$
F(x)=\sum_{n=0}^{N-1} \sum_{|i|=n} \lambda_{i} g\left(T_{i}^{-1}(x)\right)+\sum_{|i|=N} \lambda_{i} F_{N}\left(T_{i}^{-1}(x)\right)
$$

where

$$
F_{N}(x)=\sum_{n \geq 0} \sum_{|i|=n} \lambda_{i_{1}}^{N+1} \lambda_{i_{2}}^{N+2} \ldots \lambda_{i_{n}}^{N+n} g\left(T_{i}^{-1}(x)\right) .
$$

From this formula one can prove that $F$ does not satisfy the same quasi-self-similar equation in each iteration and that it has different re-normalization factors in different scales. Let us be more precise and give some points of resemblance and difference with the classical models. Recall that self-similar functions were introduced in [22] as follows.

Definition 2. A function $F: \mathbb{R}^{m} \longrightarrow \mathbb{R}$ is said to be self-affine (self-similar) of order $k \geq 0$ if there exists a bounded set $\Omega \subset \mathbb{R}^{m}$ and contractive similitudes $S_{1}, \ldots, S_{d}$ satisfying

$$
\begin{gathered}
S_{i}(\Omega) \subset \Omega, \\
S_{i}(\Omega) \cap S_{j}(\Omega)=\emptyset,
\end{gathered}
$$

there exists $\lambda_{1}, \ldots, \lambda_{d}$ such that $0<\lambda_{i}<1$ and a function $g, \mathcal{C}^{k}$ with all derivatives of order less than $k$ having fast decay satisfying

$$
F(x)=\sum_{i=0}^{d} \lambda_{i} F\left(T_{i}^{-1}(x)\right)+g(x)
$$

and finally there exists a closed subset of $\Omega$ such that $F$ is not uniformly $\mathcal{C}^{k}$ on it. 
In [22], the author proved that the multifractal formalism holds for these functions when such a function has a uniform minimal regularity. The main idea is by concentrating a self-similar measure on the singularities set which is also self-similar.

- For $N=1$, it follows from (2.3) that

$$
F(x)=\lambda_{1}^{1} F_{1}\left(T_{1}^{-1}(x)\right)+\lambda_{2}^{1} F_{1}\left(T_{2}^{-1}(x)\right)+g(x) .
$$

In general, $F_{1}$ is different from $F$. So, $F$ is not self-similar in the sense of Definition 2 .

- For $N=2$, we have

$$
\begin{aligned}
F(x) & =\lambda_{1}^{1} \lambda_{1}^{2} F_{2}\left(\left(T_{1} \circ T_{1}\right)^{-1}(x)\right)+\lambda_{1}^{1} \lambda_{2}^{2} F_{2}\left(\left(T_{1} \circ T_{2}\right)^{-1}(x)\right) \\
& +\lambda_{2}^{1} \lambda_{1}^{2} F_{2}\left(\left(T_{2} \circ T_{1}\right)^{-1}(x)\right)+\lambda_{2}^{1} \lambda_{2}^{2} F_{2}\left(\left(T_{2} \circ T_{2}\right)^{-1}(x)\right) \\
& +\lambda_{1}^{1} g\left(T_{1}^{-1}(x)\right)+\lambda_{2}^{1} g\left(T_{2}^{-1}(x)\right)+g(x) .
\end{aligned}
$$

Compared to Definition 2, $F_{1}$ disappears completely. So, the function $F$ does not satisfy the same quasi-self-similar equation in all generations.

- The non self-similarity in our case can be understood in terms of the $\lambda_{j}^{n}$. In Definition 2 these are constants, not sequences, which means that one has the same re-normalization factor in different scales. Here, we have different re-normalization factors.

- For the computation of the spectrum of singularities, the construction of Gibbs measures fails in our cases. The Gibbs measure satisfies

$$
\mu\left(\Omega_{\alpha \beta}\right) \sim \mu\left(\Omega_{\alpha}\right) \mu\left(\Omega_{\beta}\right) .
$$

However, such a construction is the main and crucial point to allow the computation of the spectrum in Definition 2. Here, for all $l, p \in \mathbb{N}^{*}$, we do not have

$$
\left|\lambda_{i_{1}}^{1} \lambda_{i_{2}}^{2} \ldots \lambda_{i_{l}}^{l}\right|\left|\lambda_{j_{1}}^{l+1} \lambda_{j_{2}}^{l+2} \ldots \lambda_{j_{p}}^{l+p}\right| \sim\left|\lambda_{i_{1}}^{1} \lambda_{i_{2}}^{2} \ldots \lambda_{i_{l}}^{l}\right|\left|\lambda_{j_{1}}^{1} \lambda_{j_{2}}^{2} \ldots \lambda_{j_{p}}^{p}\right|
$$

So the corresponding quantity $\mu\left(\Omega_{i j}\right)$ does not have the same order of magnitude as $\mu\left(\Omega_{i}\right) \mu\left(\Omega_{j}\right)$.

- By looking at the above two equalities, $N=1,2$ and Definition 2, the function $F_{1}$ which appears for $N=1$ disappears completely in the next iteration. This means that the signal does not inherit all the properties or characteristics of its original source. This can be due to natural factors such as air flow.

- The signal $F$ is written as a superposition of "slightly similar" structures at different scales, reminiscent of some possible models of turbulence.

- The $S_{j}$ may not have the same linear part. This means that the signal $F$ does not follow a fixed direction when it is propagated in space. This means that some turbulent factors take place.

- The $S_{j}$ may not have the same linear part. This means that macroscopic cells of the signal are neither of the same geometric disposition nor of the same dimensions. 
- If $F$ is $\mathcal{C}_{\rho}^{k}(x)$ for all $x \in \Omega$ then the anisotropic multifractal formalism is of no interest and this is the crucial point for imposing certain restrictions. In [12] and [22] the authors when studying the classical multifractal formalism for self-similar functions assumed that $F$ is not uniformly $\mathcal{C}^{k}$ on a closed subset of $\Omega$. In [6], [7] and [8] we considered a weak assumption. We supposed instead that $F \notin \mathcal{C}^{k}\left(x_{0}\right)$ for some point $x_{0} \in \Omega$ and we showed that this is sufficient.

- A natural assumption for our extension here is to suppose that $F \notin \mathcal{C}_{\rho}^{k}\left(x_{0}\right)$. From the anisotropic wavelet characterization in Proposition 1, this is equivalent to the fact that there exists $a_{n} \rightarrow 0, b_{n}$ and $C_{n} \rightarrow \infty$ such that

$$
\rho\left(b_{n}-x_{0}\right) \leq a_{n} \quad \text { and } \quad\left|C_{\rho, F_{N}}\left(a_{n}, b_{n}\right)\right| \geq C_{n} a_{n}^{k}, \quad \forall N .
$$

We now set down our main results in this paper. The first result gives us the global $\rho$-regularity of anisotropic quasi-self-similar functions and it is stated as follows.

Theorem 1. Let $F$ be an anisotropic quasi-self-similar function in the sense of (2.2). Then

$$
F \in \mathcal{C}_{\rho}^{\alpha_{\min }-\varepsilon}(\Omega), \quad \forall \varepsilon ; 0<\varepsilon<\alpha_{\min } .
$$

The following result computes the pointwise $\rho$-Hölder regularity for the same class of functions.

Theorem 2. Let $F$ be an anisotropic quasi-self-similar function in the sense of (2.2). We have

(i) $F$ is $\mathcal{C}_{\rho}^{k}$ in a neighborhood of $x$ for all $x \notin K$.

(ii) Let $x \in K$ and suppose that

$$
a_{\rho}(x)=\liminf _{n \rightarrow \infty} \frac{\lambda_{i_{1}(x)}^{1} \lambda_{i_{2}(x)}^{2} \cdots \lambda_{i_{n}(x)}^{n}}{\log \mu_{i_{1}(x)} \mu_{i_{2}(x)} \mu_{i_{n}(x)}}<k .
$$

Then $\alpha_{\rho, F}(x)=a_{\rho}(x)$.

For the computation of the $\rho$-spectrum of singularities, the construction of Gibbs measures fails in our case. We propose then to follow some quite similar technics as in [6] [7] and [8] with some necessary modifications due to the anisotropic homogeneous norm. Let us be more precise and explain our method. For $p, q$ real numbers define

$$
\mathcal{L}_{\rho}(p, q)=\liminf _{\varepsilon \downarrow 0}\left\{\sum_{i}\left|\lambda_{i}\right|^{p} \mid \rho\left(\Omega_{i}\right)^{-q} ; K \subset \bigcup_{i} \Omega_{i} \text { and } \rho\left(\Omega_{i}\right) \leq \varepsilon\right\} .
$$

For $n \in \mathbb{N}$ define

$$
\Theta_{\rho, n}(p, q)=\frac{1}{n} \log \sum_{|i|=n}\left|\lambda_{i}\right|^{p} \rho\left(\Omega_{i}\right)^{-q}
$$

and its limit superior

$$
\Theta_{\rho}(p, q)=\limsup _{n \rightarrow+\infty} \Theta_{\rho, n}(p, q) .
$$

One can prove the following preliminary result. 
Lemma 1. The set $\mathcal{S}=\left\{(p, q) ; \Theta_{\rho}(p, q)<0\right\}$ is convex.

This allows us to consider the separative

$$
\varphi_{\rho}(p)=\sup \left\{q ; \Theta_{\rho}(p, q)<0\right\} .
$$

We have obtained the following result

Theorem 3. Let $F$ be an anisotropic quasi-self-similar function in the sense of (2.2) and $d_{\rho}$ its $\rho$-spectrum of singularities and $\varphi_{\rho}$ the separative defined in (2.5). Then

(i) $d_{\rho}(\alpha)=-\infty$ outside $\left[\alpha_{\min }, \alpha_{\max }\right]$.

(ii) Suppose $\varphi_{\rho}$ is differentiable at $p$ and that $\alpha=\varphi_{\rho}^{\prime}(p) \in\left[\alpha_{\min }, \alpha_{\max }\right]$. Suppose further that $\mathcal{K}_{\rho}\left(p, \varphi_{\rho}(p)\right)>0$. We have

$$
d_{\rho}(\alpha)=\inf _{x}\left(\alpha x-\varphi_{\rho}(x)\right)
$$

The following result gives us the anisotropic multifractal formalism for the class of anisotropic quasi-self-similar functions.

Theorem 4. Let $F$ be an anisotropic quasi-self-similar function in the sense of (2.2) and $\eta_{\rho}$ its Besov exponent. Let $\varphi_{\rho}$ the separative defined in (2.5). We have the following implication.

$$
\text { If } \varphi_{\rho}(p)+D<k p \text { then } \eta_{\rho}(p)=\varphi_{\rho}(p)+D \text {. }
$$

\section{Global $\rho$-regularity}

In this section we shall prove Theorem 1 which gives the global $\rho$-regularity for anisotropic quasi-self-similar functions.

Proof of Theorem 1: Recall that a function $F$ is represented by means of the series

$$
F(x)=\sum_{n \geq 0} \sum_{|i|=n} \lambda_{i} g\left(T_{i}^{-1}(x)\right)
$$

According to Proposition 1, it suffices to show that

$$
\left|C_{\rho, F}(a, b)\right| \leq C a^{\alpha_{\min }-\varepsilon} .
$$

Using the Littlewood decomposition, the function $F$ can also be written as

$$
F(x)=\sum_{j \geq 0} \sum_{i \in B_{j}} \lambda_{i} g\left(T_{i}^{-1}(x)\right) .
$$

Let $a=2^{-l}, b \in \mathbb{R}^{m}$ and denote

$$
F_{i, l}^{d}(b)=\left(g \circ T_{i}^{-1}\right) * \varphi_{a}^{d}(b) .
$$


We now proceed in the following steps.

- Step 1: $0 \leq j \leq l$ and $b \in \Omega_{i}$. We then have

$$
\begin{aligned}
\left|F_{i, l}^{d}(x)\right| & =\left|\int_{\Omega_{i}}\left(g \circ T_{i}^{-1}\right)(x) \varphi_{a}^{d}(x-b) d x\right| \\
& =2^{D l} \int_{\Omega_{i}}\left(g \circ T_{i}^{-1}\right)(x) \varphi\left(A_{2^{l}}(x-b)-x^{d}\right) d x \\
& =2^{D l} \int_{\Omega_{i}}\left(g \circ T_{i}^{-1}\right)\left(x+A_{1 / 2^{l}} x^{d}\right) \varphi\left(A_{2^{l}}(x-b)\right) d x .
\end{aligned}
$$

Denote now $P_{k}^{i}$ the Taylor expansion of $g \circ T_{i}^{-1}$ to the order $k-1$ at the point $x+A_{1 / 2^{l}} x^{d}$. For the sake of simplicity we will denote this point by $x+x_{l}^{d}$. It follows that

$$
\left|F_{i, l}^{d}(x)\right|=2^{D l} \int_{\Omega_{i}}\left[\left(g \circ T_{i}^{-1}\right)\left(x+x_{l}^{d}\right)-P_{k}^{i}\left(x+x_{l}^{d}-b\right)\right] \varphi\left(A_{2^{l}}(x-b)\right) d x .
$$

Using Theorem 5 in Appendix B, we obtain

$$
\begin{aligned}
\left|F_{i, l}^{d}(x)\right| & \leq C 2^{D l} \sum_{|\sigma|=k} \int_{\Omega_{i}} \rho\left(x+x_{l}^{d}-b\right)^{d(\sigma)}\left|\varphi\left(A_{2^{l}}(x-b)\right)\right| d x \\
& \leq C 2^{D l} \sum_{|\sigma|=k} \int_{\Omega_{i}}\left(\rho\left(x_{l}^{d}\right)^{d(\sigma)}+\rho(x-b)^{d(\sigma)}\right)\left|\varphi\left(A_{2^{l}}(x-b)\right)\right| d x \\
& \leq C 2^{D l} \sum_{|\sigma|=k} 2^{-d(\sigma) l} \int_{\Omega_{i}}\left|\varphi\left(A_{2^{l}}(x-b)\right)\right| d x \\
& +C 2^{D l} \sum_{|\sigma|=k} \int_{\Omega_{i}} \rho(x-b)^{d(\sigma)}\left|\varphi\left(A_{2^{l}}(x-b)\right)\right| d x .
\end{aligned}
$$

The first part is bounded by

$$
C 2^{-k l}\left(\mu_{i}\right)^{k} \leq C 2^{k(j-l)} .
$$

The second part is bounded by

$$
\begin{aligned}
& C 2^{D l} \sum_{|\sigma|=k} \int_{\Omega_{i}}\left(L 2^{-j}\right)^{d(\sigma)}\left|\varphi\left(A_{2^{l}}(x-b)\right)\right| d x \\
\leq & C 2^{D l} L^{D k} \sum_{|\sigma|=k} 2^{-d(\sigma) l} \int_{\Omega_{i}}\left|\varphi\left(A_{2^{l}}(x-b)\right)\right| d x \\
\leq & C 2^{-k l}\left(\mu_{i}\right)^{k} \\
\leq & C 2^{k(j-l)} .
\end{aligned}
$$

It follows that

$$
\sum_{i \in B_{j}, b \in \Omega_{i}}\left|\lambda_{i} F_{i, l}^{d}(b)\right| \leq C \sum_{i \in B_{j}, b \in \Omega_{i}}\left|\lambda_{i}\right| 2^{k(j-l)}
$$


The last term is bounded by

$$
C 2^{k(j-l)} \sup _{i \in B_{j}}\left|\lambda_{i}\right| \leq C 2^{k(j-l)} 2^{-\left(\alpha_{m i n}-\varepsilon\right) j} .
$$

Whence

$$
\sum_{j \leq l} \sum_{i \in B_{j}, b \in \Omega_{i}}\left|\lambda_{i} F_{i, l}^{d}(b)\right| \leq C 2^{-\left(\alpha_{m i n}-\varepsilon\right) l} .
$$

Step 2: $0 \leq j \leq l, b \notin \Omega_{i}$. Let $\Phi$ be the wavelet such that $\Phi^{(k+1)}=\varphi$. We have

$$
\begin{aligned}
F_{i, l}^{d}(b) & =2^{D l} \int_{\Omega_{i}} g\left(T_{i}^{-1}(x)\right) \Phi^{(k+1)}\left(A_{2^{l}}(x-b)-x^{d}\right) d x \\
& =2^{D l} 2^{-(k+1) D l} \int_{\Omega_{i}}\left(g \circ T_{i}^{-1}\right)^{(k+1)}(x) \Phi\left(A_{2^{l}}(x-b)-x^{d}\right) d x .
\end{aligned}
$$

It results that for some $N$,

$$
\left|F_{i, l}^{d}(b)\right| \leq C 2^{D l} 2^{-(k+1) D l} \int_{\Omega_{i}} 2^{(k+1) j} \frac{1}{\left(1+2^{D l} \rho(x-b)\right)^{N}} d x .
$$

Now, if we design the $\rho$-distance from $b$ to $\Omega_{i}$ by

$$
\operatorname{dist}_{\rho}\left(b, \Omega_{i}\right)=\inf \left\{\rho(x-b) ; x \in \Omega_{i}\right\},
$$

we obtain

$$
\left|F_{i, l}^{d}(b)\right| \leq C 2^{D l} 2^{-(k+1) D l} \int_{\Omega_{i}} 2^{(k+1) j} \frac{1}{\left(1+2^{D l} \operatorname{dist}_{\rho}\left(b, \Omega_{i}\right)\right)^{N}} d x
$$

which implies that

$$
\left|F_{i, l}^{d}(b)\right| \leq C \frac{2^{k(j-l)}}{\left(1+2^{D l} \operatorname{dist}_{\rho}\left(b, \Omega_{i}\right)\right)^{N}}
$$

As a consequence

$$
\begin{aligned}
\sum_{i \in B_{j}, b \notin \Omega_{i}}\left|\lambda_{i} F_{i, l}^{d}(b)\right| & \leq C \sum_{i \in B_{j}, b \notin \Omega_{i}}\left|\lambda_{i}\right| \frac{2^{k(j-l)}}{\left(1+2^{D l} \operatorname{dist}_{\rho}\left(b, \Omega_{i}\right)\right)^{N}} \\
& \leq C 2^{k(j-l)} \sup _{i \in B_{j}}\left|\lambda_{i}\right| \sum_{i \in B_{j}, b \notin \Omega_{i}} \frac{1}{\left(1+2^{D l} \operatorname{dist}_{\rho}\left(b, \Omega_{i}\right)\right)^{N}} \\
& \leq C 2^{k(j-l)} 2^{-\left(\alpha_{m i n}-\varepsilon\right) j} .
\end{aligned}
$$

Whence,

$$
\sum_{j \leq l} \sum_{i \in B_{j}, b \notin \Omega_{i}}\left|\lambda_{i} F_{i, l}^{d}(b)\right| \leq C 2^{-\left(\alpha_{m i n}-\varepsilon\right) l} .
$$

It results from these steps that

$$
\sum_{j \leq l} \sum_{i \in B_{j}}\left|\lambda_{i} F_{i, l}^{d}(b)\right| \leq C 2^{-\left(\alpha_{m i n}-\varepsilon\right) l} .
$$


- Step 3: The case where $j>l$ is easier.

$$
\begin{aligned}
\sum_{i \in B_{j}}\left|\lambda_{i} F_{i, l}^{d}(b)\right| & \leq \sum_{i \in B_{j}}\left|\lambda_{i}\right| \int_{\Omega_{i}}\left|\left(g \circ T_{i}^{-1}\right)(x) \varphi_{a}^{d}(x-b)\right| d x \\
& \leq C \sup _{i \in B_{j}}\left|\lambda_{i}\right| \sum_{i \in B_{j}} \int_{\Omega_{i}}\left|\varphi_{a}^{d}(x-b)\right| d x \\
& \leq C 2^{\left(-\alpha_{\text {min }}+\varepsilon\right) j} \sum_{i \in B_{j}} \int_{\Omega_{i}}\left|\varphi_{a}^{d}(x-b)\right| d x
\end{aligned}
$$

Whence,

$$
\begin{aligned}
\sum_{j>l} \sum_{i \in B_{j}}\left|\lambda_{i} F_{i, l}^{d}(b)\right| & \leq C \sum_{j>l} 2^{\left(-\alpha_{\min }+\varepsilon\right) j} \sum_{i \in B_{j}} \int_{\Omega_{i}}\left|\varphi_{a}^{d}(x-b)\right| d x \\
& \leq C 2^{-\left(\alpha_{\min }-\varepsilon\right) l} .
\end{aligned}
$$

\section{The pointwise $\rho$-Hölder regularity}

In this section we propose to compute the pointwise $\rho$-Hölder regularity and then to prove Theorem 2. We will show that the anisotropic wavelet transform interacts well with the anisotropic contractions.

Proof of Theorem 2: The first part is obvious. Indeed, outside $K, F$ is locally a finite sum generated by $g \circ T_{i}^{-1}$. So $F$ is $\mathcal{C}_{\rho}^{k}$. Let us prove the second part. It reposes on the following Lemma which is the analog in the anisotropic homogeneous case of Lemma 2 in [6], Lemma 3.4 in [7], [8] and Lemma 3.1 in [22].

Lemma 2. For A large enough denote

$$
\Lambda_{j}(x)=\sup _{i \in B_{j}(x)}\left|\lambda_{i}\right| \quad \text { and } \quad L_{j}(x)=\sum_{l=1}^{p} \Lambda_{j, l}(x) 2^{-A(p-l)} .
$$

We have

$$
\begin{aligned}
a_{\rho}(x) & =\liminf _{j \rightarrow+\infty}\left(\inf _{i \in B_{j}(x)} \frac{\log \left|\lambda_{i}\right|}{\log \mu_{i}}\right) \\
& =\liminf _{j \rightarrow+\infty} \frac{\log \Lambda_{j}(x)}{-j \log 2}=\liminf _{j \rightarrow+\infty} \frac{\log L_{j}(x)}{-j \log 2} .
\end{aligned}
$$

Using similar decomposition as in (2.3), we obtained the following Lemma,

Lemma 3. For every $n \in \mathbb{N}$ there exists an index set $\Delta_{n}$ with finite cardinality independently of $n$ such that

$$
F(x)=\sum_{j=0}^{J-1} \sum_{i \in B_{j}} \lambda_{i} g\left(T_{i}^{-1}(x)\right)+\sum_{i \in B_{J}} \lambda_{i} F_{J}\left(T_{i}^{-1}(x)\right)
$$

where

$$
F_{J}(x)=\sum_{n=0}^{\infty} \sum_{i \in \cup_{p \in \Delta_{n}} B_{p}} \lambda_{i_{1}}^{J+1} \lambda_{i_{2}}^{J+2} \ldots \lambda_{i_{n}}^{J+n} g\left(T_{i}^{-1}(x)\right) .
$$


We now use the following result which is a crucial point in the proof and is the analog of Proposition 3 in [12] and Proposition 4.1 in [22]. In [6], [7] and [8], we did not need this result but we used direct methods.

Proposition 2. Let $x \in K, J \in \mathbb{N}$ large enough such that $\Lambda_{J}(x) \geq \frac{1}{2} L_{J}(x)$. There exists a branch $i^{0} \in B_{J}(x), b \in \Omega_{i^{0}}$ and $a \sim 2^{-J}$ such that

$$
\rho(b-x) \leq C a \quad \text { and } \quad\left|C_{\rho, F}(a, b)\right| \geq C \Lambda_{j}(x) .
$$

Because of the importance of this Proposition and for the completness of the paper, its proof will be given in Appendix A.

Let now $x \in K, J \in \mathbb{N}$ and $i^{0}$ as in Proposition 2. The Proposition means that $i^{0}$ is the branch at which the anisotropic wavelet transform is large. Now, (4.2) shows that

$$
\alpha_{\rho, F}(x) \leq a_{\rho}(x) .
$$

We now prove that $\alpha_{\rho, F}(x) \geq a_{\rho}(x)$. Let $\beta<a_{\rho}(x)$ and consider the Taylor polynomial $P_{\beta}^{i}$ of $g \circ T_{i}^{-1}$ at the point $x$ with degree $l=[\beta]$. Denote next

$$
P_{\beta}(h)=\sum_{j \geq 0} \sum_{i \in B_{j}} \lambda_{i} P_{\beta}^{i}(h) .
$$

Let $J$ be such that $2^{-J} \leq\|h\|<22^{-J}$ and $N$ the largest integer for which $x+h \in \Omega_{i(x) \mid N}$ where for an element $i \in\{1,2\}^{\mathbb{N}}$, the notation $i \mid N$ stands for the truncation of $i$ in its $N$-first entries. We have immediately $\|h\| \leq \mu_{i \mid N}$ and $x+h \notin \Omega_{i \mid N+s}$ for all $s \geq 1$. Thus,

$$
\begin{aligned}
F(x+h)-P_{\beta}(h) & =\sum_{n=0}^{N} \lambda_{i(x) \mid n}\left(g\left(T_{i}^{-1}(x+h)\right)-P_{\beta}^{i}(h)\right) \\
& -\sum_{n \geq N+1} \lambda_{i(x) \mid n} P_{\beta}^{i}(h) .
\end{aligned}
$$

Using Theorem 5 in Appendix B, we obtain that the first righthand term is bounded by

$$
C \sum_{n=0}^{N} \lambda_{i(x) \mid n} \sum_{|\sigma|=l+1, d(\sigma)>\beta} \rho(h)^{d(\sigma)} \mid \leq C \rho(h)^{\beta} .
$$

The second righthand side term is bounded by

$$
\sum_{j>J} \sum_{i \in B_{j}(x)}\left|\lambda_{i}\right| \sum_{q=0}^{l} \mu_{i}^{q}\|h\|^{q} .
$$

So it is bounded by

$$
C \sum_{j>J} 2^{-\beta j} \sum_{q=0}^{l} 2^{q j} 2^{-q J} \leq C 2^{-\beta J} .
$$

Since $\rho(h) \leq 1$, the last term is bounded by

$$
C\|h\|^{\beta} \leq C \rho(h)^{\beta} .
$$

As a consequence

$$
\left|F(x+h)-P_{\beta}(h)\right| \leq C \rho(h)^{\beta} .
$$




\section{The $\rho$-spectrum of singularities}

To compute the $\rho$-spectrum of singularities, it is sufficient to construct a Borel probability measure supported by the set of singularities. In [12] and [22], the idea was by constructing Gibbs measures. In [6], [7], [8] and in the present work, such a contruction is not possible. Indeed, in [12] and [22], the obtained measure was defined by

$$
\mu\left(\Omega_{i}\right)=\lambda_{i} \text { and } 0 \text { elswhere. }
$$

Remark in this case that for $i=\left(i_{1}, i_{2}, \ldots, i_{n}\right)$ and $j=\left(j_{1}, j_{2}, \ldots, j_{l}\right)$ two multi-indices one has

$$
\mu\left(\Omega_{i j}\right)=\lambda_{i j}=\lambda_{i} \lambda_{j}=\mu\left(\Omega_{i}\right) \mu\left(\Omega_{j}\right) .
$$

This means that $\mu$ is a strong multiplicative Gibbs or Bernouilli measure. In such a situation, the multifractal formalism is an obvious application of the result of Brown, Michon and Peyrière in [16]. In [6], [7], [8] and in the present work, the situation is different. The two quantities have not the same order of magnitude because for all $p, q \in \mathbb{N}^{*}$, the quantity $\left|\lambda_{i_{1}}^{1}\right| \ldots\left|\lambda_{i_{p}}^{p}\right| \cdot\left|\lambda_{j_{1}}^{p+1}\right| \ldots\left|\lambda_{j_{q}}^{p+q}\right|$ has not the same order of magnitude as $\left|\lambda_{i_{1}}^{1}\right| \ldots\left|\lambda_{i_{p}}^{p}\right| \cdot\left|\lambda_{j_{1}}^{1}\right| \ldots\left|\lambda_{j_{q}}^{q}\right|$. For this reason, we have introduced new approaches for the spectrum of singularities in [6], [7] and [8]. Our idea was based on using Frostmann's method and we applied the results of [11]. In the present work, the method is quite similar. We will introduce suitable modifications on the Frostmann's method and to the techniques of [11] to prove our result. Consider a Borel probability measure $\mu$ on $\Omega$ and a sequence of partitions $\left(\mathcal{F}_{n}\right)_{n}$ of $\Omega$ such that

- $\mathcal{F}_{n+1}$ is a refinement of $\mathcal{F}_{n}$.

- For $x \in \Omega$, let $\Omega_{n}(x) \in \mathcal{F}_{n}$ that contains $x$. Then $\rho\left(\Omega_{n}(x)\right) \rightarrow 0$ as $n \rightarrow \infty$.

- $\sup _{U \in \mathcal{F}_{n}} \rho(U) \rightarrow 0$ as $n \rightarrow \infty$.

Denote $\mathcal{F}=\bigcup_{n} \mathcal{F}_{n}$

Definition 3. $\zeta: \mathcal{F} \rightarrow \mathbb{R}_{+}$is said to be a $\rho$-Frostmann function on $\Omega$ if 0 is adherent to the sequence $\left(\sup _{U \in \mathcal{F}} \zeta(U)\right)_{n}$ and if $\mathcal{H}_{\rho}(\zeta)>0$ where

$$
\mathcal{H}_{\rho}(\zeta)=\liminf _{\varepsilon \downarrow 0}\left\{\sum_{s} \zeta\left(U_{s}\right) ;\left\{U_{s}\right\} \varepsilon-\rho-\text { covering of } \Omega\right\} .
$$

We have the following Lemma which is the analog of Frostmann's one.

Lemma 4. Let $\zeta$ be a $\rho$-Frostmann function. There exists a probability measure $\widetilde{\zeta}$ on $\Omega$ and constants $C, \varepsilon>0$ such that

$$
\widetilde{\zeta}(U) \leq C \zeta(U), \quad \forall U \in \mathcal{F}, \rho(U) \leq \varepsilon .
$$


For $p, q$ real numbers define

$$
\mathcal{L}_{\rho}(p, q)=\lim _{\varepsilon \downarrow 0} \inf \left\{\left.\sum_{s}^{*} \mu\left(U_{s}\right)^{p} \rho\left(U_{s}\right)\right|^{-q} ; U_{s} \in \mathcal{F}_{n} \text { and } \rho\left(U_{s} \mid \leq \varepsilon\right\}\right.
$$

and

$$
\Theta_{\rho, n}(p, q)=\frac{1}{n} \log \sum_{U \in \mathcal{F}_{n}}^{*} \mu(U)^{p} \rho(U)^{-q}
$$

where $*$ designs that we restrict only on sets with non zero measure. Let

$$
\varphi_{\rho}(p)=\sup \left\{y ; \Theta_{\rho}(p, q)=\limsup _{n \rightarrow+\infty} \Theta_{\rho, n}(p, q)<0\right\} .
$$

Finally, we suppose that $\varphi_{\rho}$ is of finite values on an open interval in $\mathbb{R}$. Consider

$$
E_{\alpha}=\left\{x ; \liminf _{n \rightarrow+\infty} \frac{\log \mu\left(\Omega_{n}(x)\right)}{\log \rho\left(\Omega_{n}(x)\right)}=\alpha\right\}
$$

Proof of Theorem 3: The first point is obvious since in this case the set $E(\alpha)=\emptyset$. We then prove the second. In our situation, we set

$$
\mathcal{F}_{n}=\left\{\Omega_{i} ;|i|=n\right\}
$$

and we consider

$$
\mu(U)=\left\{\begin{array}{cll}
\left|\lambda_{i}\right| & \text { if } & U=\Omega_{i}, \\
0 & \text { if } & \text { not. }
\end{array}\right.
$$

The function $\zeta$ will be defined by

$$
\zeta(U)=\left\{\begin{array}{cll}
\left|\lambda_{i}\right|^{p} \rho\left(\Omega_{i}\right)^{-\varphi_{\rho}(p)} & \text { if } & U=\Omega_{i} \\
0 & \text { if } & \text { not. }
\end{array}\right.
$$

Denote $\alpha=\varphi_{\rho}^{\prime}(p)$ and consider the sets

$$
\begin{aligned}
& U_{\alpha}=\left\{x ; \lim _{n \rightarrow+\infty} \frac{\log \left|\lambda_{i}\right|}{\log \rho\left(\Omega_{i}\right)}=\alpha\right\}, \\
& V_{\alpha}=\left\{x ; \liminf _{n \rightarrow+\infty} \frac{\log \left|\lambda_{i}\right|}{\log \rho\left(\Omega_{i}\right)} \leq \alpha\right\}
\end{aligned}
$$

and

$$
\widetilde{V}_{\alpha}=\left\{x ; \liminf _{n \rightarrow+\infty} \frac{\log \widetilde{\zeta}\left(\Omega_{i}\right)}{\log \rho\left(\Omega_{i}\right)} \geq \alpha p-\varphi_{\rho}(p)\right\} .
$$

Lemma 4 yields that $U_{\alpha} \subset \widetilde{V}_{\alpha}$. On the other hand, Billingsley Theorem implies that

$$
\operatorname{dim}_{\rho} U_{\alpha} \geq \alpha p-\varphi_{\rho}(p) .
$$


Whence,

$$
d_{\rho}(\alpha) \geq \alpha p-\varphi_{\rho}(p)
$$

We now prove the converse. Consider the set

$$
\widehat{V}_{\alpha}=\left\{x ; \limsup _{n \rightarrow+\infty} \frac{\log \widetilde{\zeta}\left(\Omega_{i}\right)}{\log \rho\left(\Omega_{i}\right)} \geq \alpha p-\varphi_{\rho}(p)\right\} .
$$

Let $\varepsilon>0$ and $\delta=\delta_{\varepsilon}>0$ be such that $C_{\rho}\left(p+\varepsilon, \varphi_{\rho}(p)\right)<-\delta<0$. For $n$ large, this implies that

$$
\sum_{n} \mu\left(\Omega_{n}\right)^{p+\varepsilon} \rho\left(\Omega_{n}\right)^{-\varphi_{\rho}(p)}<e^{-n \delta} .
$$

On the other hand, for $n$ large we have $\mu\left(\Omega_{n}\right) \geq \rho\left(\Omega_{n}\right)^{\alpha+\eta}$ for some $\eta>0$ small enough. So, it results that

$$
\mathcal{H}_{\rho}^{\gamma}\left(\widehat{V}_{\alpha}\right)<\infty \quad \text { with } \quad \gamma=(\alpha+\eta)(p+\varepsilon)-\varphi_{\rho}(p) .
$$

Since, $\varepsilon$ and $\eta$ are chosen small enough, it follows that

$$
\operatorname{dim}_{\rho} \widehat{V}_{\alpha} \leq \alpha p-\varphi_{\rho}(p) .
$$

Hence,

$$
d_{\rho}(\alpha) \leq \alpha p-\varphi_{\rho}(p) .
$$

Finally, equations (5.1) and (5.2) yield that

$$
d_{\rho}(\alpha)=\alpha p-\varphi_{\rho}(p) .
$$

\section{The group dilation multifractal formalism}

In this section we shall prove the validity of the anisotropic multifractal formalism for the class of anisotropic quasi-self-similar functions. We will show that the homogeneous Besov exponent can be computed by means of $\varphi_{\rho}$. We will prove precisely that

$$
\eta_{\rho}(p)=D+\varphi_{\rho}
$$

Since we have proved in the previous section that

$$
d_{\rho}(\alpha)=\inf _{p}\left(\alpha p+\varphi_{\rho}(p)\right),
$$

we obtain

$$
d_{\rho}(\alpha)=\inf _{p}\left(\alpha p-\eta_{\rho}(p)+D\right)
$$

which is the desired formula.

Proof of Theorem 4: Recall that

$$
C_{\rho, F}(a, b)=\int F(t) \varphi_{a, b}^{d}(t) d t .
$$


We now consider its partial derivatives in space. We have

$$
\frac{\partial C_{\rho, F}(a, b)}{\partial b}=-\frac{1}{a^{d}} \widetilde{C}_{\rho, F}(a, b)
$$

where we adopted the following notations

$$
\begin{gathered}
\frac{1}{a^{d}}=\left(\frac{1}{a^{d_{1}}}, \frac{1}{a^{d_{2}}}, \ldots, \frac{1}{a^{d_{m}}}\right) \in \mathbb{R}_{+}^{m}, \\
\widetilde{C}_{\rho, F}(a, b)=\left(\widetilde{C}_{\rho, F}^{1}(a, b), \widetilde{C}_{\rho, F}^{2}(a, b), \ldots, \widetilde{C}_{\rho, F}^{m}(a, b)\right)
\end{gathered}
$$

and where for $i=1,2, \ldots, m, \widetilde{C}_{\rho, F}^{i}(a, b)$ is the wavelet transform of $F$ relatively to $\frac{\partial \varphi^{d}}{\partial x_{i}}$. Similarly the derivation according to the scale $a$ gives

$$
\frac{\partial C_{\rho, F}(a, b)}{\partial a}=-\frac{D}{a} C_{\rho, F}(a, b)-\frac{1}{a} \widehat{C}_{\rho, F}(a, b)
$$

where

$$
\widehat{C}_{\rho, F}(a, b)=\frac{1}{a^{D}} \int F(t)<\tilde{A}_{a}(t-b), \nabla \varphi^{d}\left(A_{a}^{-1}(t-b)\right)>d t
$$

and

$$
\tilde{A}_{a}=\operatorname{diag}\left(\frac{d_{1}}{a^{d_{1}}}, \frac{d_{2}}{a^{d_{2}}}, \ldots, \frac{d_{m}}{a^{d_{m}}}\right) .
$$

Let now, $A_{j}=\left[2^{-j-1}, 2^{-j}\right], i \in B_{j}$ be such that $\left|C_{\rho, F}(a, b)\right| \geq C\left|\lambda_{i}\right|$ and denote

$$
\Gamma_{j}(p)=\int_{A_{j} \times \mathbb{R}^{m}}\left|C_{\rho, F}(a, b)\right|^{p} d a d b
$$

We have on one hand

$$
\begin{aligned}
\Gamma_{j}(p) & \geq C \sum_{i \in B_{j}} \int_{A_{j} \times \Omega_{i}}\left|C_{\rho, F}(a, b)\right|^{p} d a d b \\
& \geq C \sum_{i \in B_{j}}\left|\lambda_{i}\right|^{p} 2^{-j} 2^{-D j} .
\end{aligned}
$$

On the other hand

$$
\begin{aligned}
\Gamma_{j}(p) \leq C \sum_{i \in B_{j}} \int_{A_{j} \times \Omega_{i}}\left|C_{\rho, F}(a, b)\right|^{p} d a d b \\
+C \sum_{i \notin B_{j}} \int_{A_{j} \times \Omega_{i}}\left|C_{\rho, F}(a, b)\right|^{p} d a d b .
\end{aligned}
$$

The first right hand side term in the last inequality above is bounded by

$$
C \sum_{i \in B_{j}}\left|\lambda_{i}\right|^{p} 2^{-j} 2^{-D j}
$$


It remains to estimate the second term which will be denoted by

$$
X_{j}(p)=X_{j}^{1}(p)+X_{j}^{2}(p)
$$

where

$$
X_{j}^{1}(p)=\sum_{i, \mu_{i}<2^{-j}} \int_{A_{j} \times \Omega_{i}}\left|C_{\rho, F}(a, b)\right|^{p} d a d b
$$

and

$$
X_{j}^{2}(p)=\sum_{i, \mu_{i} \geq 22^{-j}} \int_{A_{j} \times \Omega_{i}}\left|C_{\rho, F}(a, b)\right|^{p} d a d b .
$$

We have

$$
X_{j}^{1}(p) \leq C \sum_{n \geq j+1} \sum_{i \in B_{n}}\left|\lambda_{i}\right|^{p} 2^{-n} 2^{-D n}
$$

and

$$
X_{j}^{2}(p) \leq C \sum_{n \leq j-1} \sum_{i \in B_{n}}\left|\lambda_{i}\right|^{p} 2^{-n} 2^{-D n} .
$$

Now recall that $C_{\rho}\left(p, \varphi_{\rho}(p)\right)=0$ which means that for $n$ large enough

$$
\sum_{|i|=n}\left|\lambda_{i}\right|^{p} \rho\left(\Omega_{i}\right)^{-\varphi_{\rho}(p)}=e^{o(1 / n)} .
$$

As a consequence,

$$
\begin{aligned}
X_{j}^{1}(p) & \leq C \sum_{n \geq j+1} 2^{-n} n e^{o(1 / n)} 2^{-n\left(D+\varphi_{\rho}(p)\right)} \\
& \leq C j 2^{-j} e^{o(1 / j)} 2^{-j\left(D+\varphi_{\rho}(p)\right)}
\end{aligned}
$$

and similarly

$$
\begin{aligned}
X_{j}^{2}(p) & \leq C \sum_{n \geq j+1} 2^{-n} e^{o(1 / n)} 2^{-n\left(D+\varphi_{\rho}(p)\right)} \\
& \leq C 2^{-j} e^{o(1 / j)} 2^{-j\left(D+\varphi_{\rho}(p)\right)}
\end{aligned}
$$

Hence,

$$
X_{j}(p) \leq C j 2^{-j} e^{o(1 / j)} 2^{-j\left(D+\varphi_{\rho}(p)\right)} .
$$

On the other side, there exists constants $C_{1}, C_{2}>0$ such that for $j$ large

$$
C_{1} e^{o(1 / j)} \leq \sum_{i \in B_{j}}\left|\lambda_{i}\right|^{p} \rho\left(\Omega_{i}\right)^{-\varphi_{\rho}(p)} \leq C_{2} j e^{o(1 / j)} .
$$

So that

$$
C_{1} 2^{-j} e^{o(1 / j)} 2^{-j\left(D+\varphi_{\rho}(p)\right)} \leq \sum_{i \in B_{j}}\left|\lambda_{i}\right|^{p} 2^{-j} 2^{-D j} \leq C_{2} j 2^{-j} e^{o(1 / j)} 2^{-j\left(D+\varphi_{\rho}(p)\right)} .
$$

From all these estimates we conclude that

$$
C 2^{-j} e^{o(1 / j)} 2^{-j\left(D+\varphi_{\rho}(p)\right)} \leq \Gamma_{j}(p) \leq C^{\prime} j 2^{-j} e^{o(1 / j)} 2^{-j\left(D+\varphi_{\rho}(p)\right)} .
$$


As a result

$$
\limsup _{a \rightarrow 0} a^{-\left(D+\varphi_{\rho}(p)\right)} e^{o(1 / \log a)} \int_{\mathbb{R}^{m}}\left|C_{\rho, F}(a, b)\right|^{p} d b \geq C>0
$$

and

$$
\limsup _{a \rightarrow 0} a^{-\left(D+\varphi_{\rho}(p)\right)} \frac{e^{o(1 / \log a)}}{\log a} \int_{\mathbb{R}^{m}}\left|C_{\rho, F}(a, b)\right|^{p} d b \leq C^{\prime}<+\infty .
$$

Hence,

$$
\eta_{\rho}(p)=D+\varphi_{\rho}(p)
$$

\section{Appendix A: Proof of Proposition 2[12]}

Consider $i^{0} \in B_{J}(x)$ such that $\Lambda_{J}(x)$ is reached. Let $a_{n}, b_{n}$ as in assumption (2.4) and take $b=T_{i^{0}}\left(b_{n}\right)$ and $a=a_{n} \mu_{i^{0}}^{D}$. So the condition $\rho(b-x) \leq C a$ is immediate. Let us prove the rest of the Proposition. Denote

$$
r_{i}=\frac{a}{\mu_{i}} \quad \text { and } \quad \chi_{i}=T_{i}^{-1}(b) .
$$

Using the Littlewood decomposition of $F$ in Lemma 3, we obtain

$$
\begin{aligned}
C_{\rho, F}(a, b) & =\sum_{j=0}^{J-1} \sum_{i \in B_{j}} \lambda_{i} \int_{\Omega_{i}} g(t) \varphi_{r_{i}, \chi_{i}}^{d}(t) d t \\
& +\sum_{i \in B_{J}, i \neq i^{0}} \lambda_{i} \int_{\Omega_{i}} F_{J}(t) \varphi_{r_{i}, \chi_{i}}^{d}(t) d t \\
& +\lambda_{i^{0}} \int_{\Omega_{i^{0}}} F_{J}(t) \varphi_{r_{i^{0}}, \chi_{i} 0}^{d}(t) d t .
\end{aligned}
$$

Denote the terms in the right hand side by $X, Y$ and $Z$ respectively. We have the following estimates.

$$
\begin{aligned}
|Z| & =\left|\lambda_{i^{0}}\right|\left|C_{\rho, F_{J}}\left(r_{i^{0}}, \chi_{i^{0}}\right)\right| \\
& \geq\left|\lambda_{i^{0}}\right| C_{n} a_{n}^{k} \\
& \geq\left|\lambda_{i^{0}}\right| C_{n}\left(\frac{a}{\mu_{i^{0}}}\right)^{k} .
\end{aligned}
$$

Now, from the fact that $i^{0} \in B_{J}(x), 2^{-J} \leq \mu_{i^{0}} \leq 22^{-J}$. With the fact that $a \sim 2^{-J}$ we obtain $\frac{a}{\mu_{i^{0}}} \sim C t e \geq \frac{1}{2}$. Hence,

$$
|Z| \geq \frac{C_{n}}{2} \Lambda_{J}(x)
$$


We now estimate the term $X$. Consider the Taylor expansion of $g$ at the order $k-1$. For $0 \leq j \leq J-1$ and $i \in B_{j}$ we have

$$
\begin{aligned}
\left|C_{\rho, g}\left(r_{i}, \chi_{i}\right)\right| & =\left|\int\left[g(t)-P_{k-1}\left(t-\chi_{i}\right)\right] \varphi_{r_{i}, \chi_{i}}^{d}(t)\right| d t \\
& \leq C \sum_{|\sigma|=k} \int \rho\left(t-\chi_{i}\right)^{d(\sigma)}\left|\varphi_{r_{i}, \chi_{i}}^{d}(t)\right| d t \\
& \leq C \sum_{|\sigma|=k} r_{i}^{d(\sigma)} \int \rho(t)^{d(\sigma)}\left|\varphi^{d}(t)\right| d t \\
& \leq C \sum_{|\sigma|=k} 2^{-J d(\sigma)} 2^{j d(\sigma)} .
\end{aligned}
$$

As a consequence,

$$
|X| \leq C \Lambda_{J}(x) .
$$

Finally, the term $Y$ is bounded by

$$
C \sum_{i \in B_{J}, i \neq i^{0}}\left|\lambda_{i}\right| \int\left|\varphi_{r_{i}, \chi_{i}}^{d}(t)\right| d t .
$$

Hence,

$$
|Y| \leq C \Lambda_{J}(x) .
$$

It results from estimates (7.1), (7.2) and (7.3) for $n$ large enough that

$$
C_{\rho, F}(a, b) \geq C \Lambda_{J}(x) .
$$

\section{Appendix B}

Recall that the homogeneous norm was defined on $\mathbb{R}^{m}$ by

$$
\rho(x)=\left\{\begin{array}{cc}
r(x) & , \quad x \neq 0, \\
0, & x=0 .
\end{array}\right.
$$

where $r(x)$ is the unique value of $r$ such that $\varphi_{x}(r)=1$. The following result gives some basic properties of $\rho$.

Lemma 5. There exists positive constants $C_{1}, C_{2}$ and $C_{3}$ such that

$$
\begin{gathered}
C_{1}\|x\| \leq \rho(x) \leq C_{2}\|x\|^{1 / d_{m}} \quad \text { whenever } \quad \rho(x) \leq 1 . \\
C_{1}^{1 / d_{m}}\|x\|^{1 / d_{m}} \leq \rho(x) \leq C_{2}^{d_{m}}\|x\| \quad \text { whenever } \quad \rho(x) \geq 1 . \\
\rho(x+y) \leq C_{3}(\rho(x)+\rho(y)) \quad \forall x, y .
\end{gathered}
$$

The following Theorem is an homogeneous version of Taylor's expansion. 
Theorem 5. Let $\Delta$ be the additive semi-group of $\mathbb{R}$ generated by $0, d_{1}, \ldots, d_{m}$. Let $\delta \in \Delta$ positive and $k=[\delta]$. Let $F$ be a $\mathcal{C}^{k+1}$ function on $\mathbb{R}^{m}$. There exists constants $C_{1}$ and $C_{2}$ (depending eventually on $\delta$ and $F$ ) such that

$$
\left|F(x+y)-P_{x}(y)\right| \leq C_{1} \sum_{|\sigma|=k+1, d(\sigma)>\delta} \rho(y)^{d(\sigma)} \sup _{\rho(h) \leq C_{2}^{k+1} \rho(y)}\left|\partial^{\sigma} F(x+h)\right| .
$$

where $P_{x}$ is the homogeneous Taylor polynomial of order $k$ of $F$ at $x$.

\section{Acknowledgment}

The author thanks the referee(s) for his/her/their comments, which greatly helped improve the presentation and the quality of the paper.

\section{References}

[1] A. Adrover, W. Schwalm, M. Giona and D. Bachand, Scaling and scaling crossover for transport on anisotropic fractal structures. Phys. Rev. E, 55(6) (1997), 7304-7312.

[2] S. T. Ali, J. P. Antoine and J. P. Gazeau, Coherent states, wavelets and their generalizations. Graduate texts in contemporary physics, Springer 2000.

[3] A. Arneodo, E. Bacry and J. F. Muzy, Singularity spectrum of fractal signals: exact results. Journal of Statistical Physics, 70(3/4) (1993), 635-674.

[4] P. Audet and J. C. Mareschal, Wavelet analysis of the coherence between Bouguer gravity and topography: application to the elastic thickness anisotropy in the Canadian Shield. Geophys. J. Int. (2007) 168, 287-298.

[5] R. C. Ball, G. Caldarelli and A. Flammini, Angular structure of lacunarity and the renormalization group. Phys. Rev. Letters, 85(24) (2000), 5134-5137.

[6] A. Ben Mabrouk, Multifractal analysis of some non isotropic quasi-self-similar functions. FJDS, 7(1) (2005), 23-63.

[7] A. Ben Mabrouk, Wavelet analysis of anisotropic quasi-self-similar functions in a nonlinear case. Colloque WavE 2006, Federal Polytechnic School of Lausanne, Switzerland, $10^{\text {th }}$ to $14^{\text {th }}$ of July 2006 .

[8] A. Ben Mabrouk, On some nonlinear non isotropic quasi-self-similar functions. Nonlinear Dynamics, 51 (2008), 379-398.

[9] A. Ben Mabrouk, Study of some nonlinear self-similar distributions. International Journal of Wavelets, Multiresolution and Information Processing, 5(6) (2007), 907916.

[10] A. Ben Mabrouk, Wavelet analysis of nonlinear self-similar distributions with oscillating singularity. International Journal of Wavelets, Multiresolution and Information Processing. Accepted November 24, 2007. 
[11] F. Ben Nasr, Analyse multifractale de mesures. CRAS. Paris 319(I) (1994), 807-810.

[12] M. Ben Slimane. Etude du formalisme multifractal pour les fonctions. Thèse de Mathématiques. Ecole normale supérieure des Ponts et Chaussées (1996).

[13] D. Blackmore and G. Zhou, A new fractal model for anisotropic surfaces. Int. J, Mach. Tools Manufact., 38(5-6) (1998), 551-557.

[14] D. Blackmore and G. Zhou, Fractal analysis of height distributions of anisotropic rough surfaces, Fractals 6 (1998), 43-58.

[15] M. Bownik and K. P. Ho, Atomic and molecular decomposition of anisotropic TriebelLizorkin spaces. Trans. Amer. Math. Soc. 358(4) (2005), 1469-1510.

[16] G. Brown, G. Michon and J. Peyrière, On the multifractal analysis of measures. Jour. Stat. Phys, 66(1992), 775-779.

[17] M. J. Fouch, P. G. Silver, D. R. Bell and J. N. Lee, Small-scale variations in seismic anisotropy near Kimberley, South Africa, Geophys. J. Int., 157 (2004), 764-774.

[18] U. Frisch and G. Parisi, Fully developped turbulence and intermittency. Proc. Int. Summer school Phys. Enrico Fermi, North Holland, p. 84-88, 1985.

[19] K. P. Ho, Anisotropic function spaces, PhD. Dessertation Washington university 2002.

[20] S. Hofmann, X. Li and D. Yang, A generalized characterization of commutators of parabolic singular integrals. Canad. Math. Bull. 42(4)(1999), 463-477.

[21] G. Iovane, Cantorian space-time, Fantappie's final group, accelerated universe and other consequences, Chaos, Solitons and Fractals 27 (2006) 618-629.

[22] S. Jaffard, Multifractal formalism for functions. Part 2: selfsimilar functions. SIAM Journal Math. Anal. 28(4) (1997), 971-998.

[23] J. F. Kirby and C. J. Swaina, Mapping the mechanical anisotropy of the lithosphere using a 2D wavelet coherence, and its application to Australia, Phys. of The Earth and Planetary Interiors, 158(2-4) (2006), 122-138.

[24] G. Kyriazis, Multilevel characterization of anisotropic function spaces. SIAM J. Math. Anal. 36(2) (2004), 441-462.

[25] S. Lovejoy and D. Schertzer, Scale invariance, symmetries, fractals and stochastic simulations of atmospheric phenomena, Bulletin of the American Meteorological Society, 6(1) (1986), 21-32.

[26] M. J. Fouch, David R. Bell , Paul G. Silver and Jean N. Lee, Small-scale variations in seismic anisotropy near Kimberley, South Africa, surface of the earth: Global studies, 2006 IRIS 5-year proposal.

[27] F. G. Silver, S. S. Gao, K. H. Liu, and the Kaapvaal seismic group, mantle deformation beneath southern Africa. Geophys. Resear. Letters, 28 (2001), 24932496. 
[28] H. Triebel, Wavelet bases in anisotropic function spaces. Preprint.

[29] Y. Wang and J. C. Mareschal, Elastic thickness of the lithosphere in the central canadian shield. Geophys. Resear. Letters, 26(19) (1999), 3033-3035. 